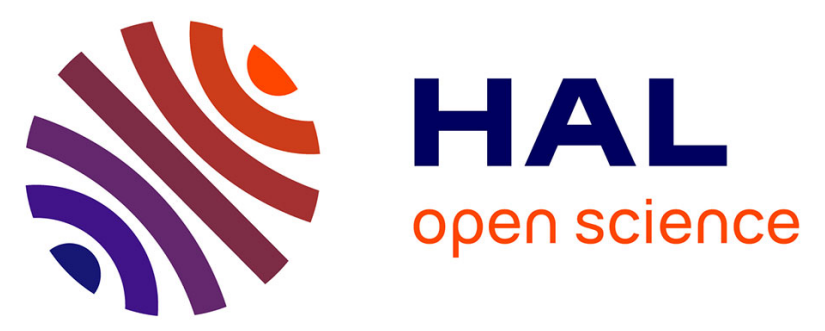

\title{
Nonlinear response of a gallium phosphide nanopatterned photonic waveguide in the $\mathrm{CW}$ regime
}

Weilin Xie, Aude Martin, Gregoire Beaudoin, Isabelle Sagnes, Fabien

Bretenaker, Sylvain Combrié, Fabrice Raineri, Alfredo De Rossi

\section{- To cite this version:}

Weilin Xie, Aude Martin, Gregoire Beaudoin, Isabelle Sagnes, Fabien Bretenaker, et al.. Nonlinear response of a gallium phosphide nanopatterned photonic waveguide in the $\mathrm{CW}$ regime. Optics Letters, 2019, 44 (11), pp.2823. 10.1364/OL.44.002823 . hal-02139737

\section{HAL Id: hal-02139737 \\ https://hal.science/hal-02139737}

Submitted on 22 Oct 2019

HAL is a multi-disciplinary open access archive for the deposit and dissemination of scientific research documents, whether they are published or not. The documents may come from teaching and research institutions in France or abroad, or from public or private research centers.
L'archive ouverte pluridisciplinaire HAL, est destinée au dépôt et à la diffusion de documents scientifiques de niveau recherche, publiés ou non, émanant des établissements d'enseignement et de recherche français ou étrangers, des laboratoires publics ou privés. 


\title{
Nonlinear response of a Gallium Phosphide Nanopatterned Photonic Waveguide in the CW regime
}

\author{
Weilin Xie ${ }^{3,4}$, Aude Martin ${ }^{1,2}$, Gregoire Beaudoin ${ }^{2}$, Isabelle Sagnes ${ }^{2}$, Fabien BretenakeR ${ }^{3}$, \\ Sylvain Combrié ${ }^{1}$, Fabrice Raineri ${ }^{2}$, ANd Alfredo De Rossi ${ }^{1, *}$ \\ ${ }^{1}$ Thales Research and Technology, CNRS, Université Pasris-Sud, Université Paris-Saclay, Campus Polytechnique, 91767 Palaiseau Cedex, France \\ ${ }^{2}$ Centre de Nanosciences et de Nanotechnologies, C2N UMR 9001, CNRS, Université Paris-Sud, Université Paris-Saclay, 91120 Palaiseau, France \\ ${ }^{3}$ Laboratoire Aimé Cotton, CNRS, Université Paris-Sud, Université Paris-Saclay, Campus d'Orsay, 91405 Orsay Cedex, France \\ ${ }^{4}$ School of Optics and Photonics, Beijing Institute of Technology, 100081, Beijing, China \\ *Corresponding author:wlxie@bit.edu.cn
}

Compiled May 7, 2019

The third-order Kerr nonlinear response in Gallium Phosphide nanoscale waveguides is measured through continuous-wave four-wave mixing. The extracted nonlinear coefficient ranges from about $800 \mathrm{~W}^{-1} \mathrm{~m}^{-1}$ to $1400 \mathbf{W}^{-1} \mathbf{m}^{-1}$, consistently with an estimated material nonlinearity $n_{2}=3.5 \times 10^{-18} \mathbf{W}^{-1} \mathbf{m}^{2}$. The roles of the residual absorption and the related thermal effects are discussed. () 2019 Optical Society of America

http://dx.doi.org/10.1364/XX.XX.XXXXXX

Nanoscale semiconductor photonic structures are subjected to large optical power density in order to achieve efficient nonlinear operation, which is required for practical implementation of compact devices for all-optical signal processing [1,2] and novel lightwave sources such as integrated parametric oscillators $[3,4]$. While semiconductors, owing to their large refractive index, are suitable for dense photonic integration, they also suffer from nonlinear absorption [5], which depends on the electronic bandgap compared to the photon energy [6]. The resulting heat generated in such tiny volumes may result in severe thermal effects which can disrupt the operation of the device. This effect becomes critical in suspended nanophotonic structures such as photonic crystal cavities (e.g. optical memories [7]) and waveguides, where heat can only flow through the thin structure towards the anchoring points.

In this respect, Gallium Phosphide $(\mathrm{GaP})$ is a semiconductor material endowed with many attractive features: first, as Gallium Arsenide, its zinc-blende cristalline structure results in sizeable second order nonlinearity and piezolectric effect. In contrast, its much larger electronic gap $(2.4 \mathrm{eV})$ implies that two-photon absorption vanishes in telecom spectral band [8] and also three photon absorption is small. Moreover, its thermal conductivity is $110 \mathrm{~W} \mathrm{~K}^{-1} \mathrm{~m}^{-1}$, about 20 times larger than in InGaP, a material which we have shown to be otherwise very appropriate for nonlinear optics [9]. GaP ring resonator [10], microdisk [11, 12], and cavities [13], have been considered for their large $\chi^{(2)}$ nonlinearity. GaP photonic wires on insulator have recently been developed and have been shown to allow low power optical frequency combs [14]. Here we focus on suspended photonic crystal ( $\mathrm{PhC}$ ) waveguides, as the large surface to volume ratio and peculiar optical confinement allows, in principle, effects related to the surface and, therefore, justify specific measurements of nonlinear and linear parameters on this platform. In particular, we measure the residual absorption which we attribute to surface defects. The ultra-fast nonlinear response due to the optical Kerr effect has been addressed in the pulsed regime [15]. Here we address the continuous-wave (CW) regime, where average power is larger. We measure the nonlinear parametric coefficient through four-wave mixing (FWM) with detunings large enough $(>100 \mathrm{GHz})$ to rule out the contributions from slower effects, such as those which would result from the excitation of free-carriers [16].

The nonlinear waveguide is made out of a $230 \mathrm{~nm}$ thick $\mathrm{GaP}$ slab on top of $1 \mu \mathrm{m}$ thick sacrificial layer of $A l_{0.7} G a_{0.3} P$, grown on a GaP substrate by metal-organic chemical vapor deposition (MOCVD). After patterning with electron-beam lithography and inductively coupled plasma (ICP) reactive ion etching, the suspended membrane (Fig.1(c)) is obtained after the removal of the sacrificial layer through critical dry etching. The sample is accessed (Fig.1(a)) through lensed fiber (beam waist $1 / e^{2}$ is $\approx 2.5 \mu \mathrm{m}$ ) or microscope objectives (N.A. 0.95 ). In both configurations, on-chip mode adapters are used to reduce coupling losses to less than $5 \mathrm{~dB}$ per side, including objective losses [17]. The waveguide is $2.5 \mathrm{~mm}$ long and consists of three different sections. The main section, $2 \mathrm{~mm}$ long, is the core, designed such that in the range between 1550 and $1570 \mathrm{~nm}$ light propagates in the so-called slow-light (SL) regime [18], where the group index $n_{g}>10$ becomes larger than the material index ( 3.07 at $\left.1.55 \mu \mathrm{m}\right)$. On both sides, a transition region consisting of a tapered (T) and an input (I) sections, in order to adiabatically transform and accelerate/decelerate the mode before reaching the mode adapters to the free space. The design of the PhC waveguide consists in a triangular lattice of air holes (period $a=445 \mathrm{~nm}$ and radii $r=0.228 a$ ) and a line defect (width $w=718 n m=\sqrt{3} a$ ). The dispersion is controlled by shifting the first row of airholes towards the line defect by $s=72 \mathrm{~nm}$. In the input section $w=860 \mathrm{~nm}$ and $s=36 \mathrm{~nm}$ while in the taper (T) $w=780 \mathrm{~nm}$ and $s=56 \mathrm{~nm}$.

The characterization is based on a suitable implementation of the optical coherence tomography (OCT) [19-21] where the spec- 
a)

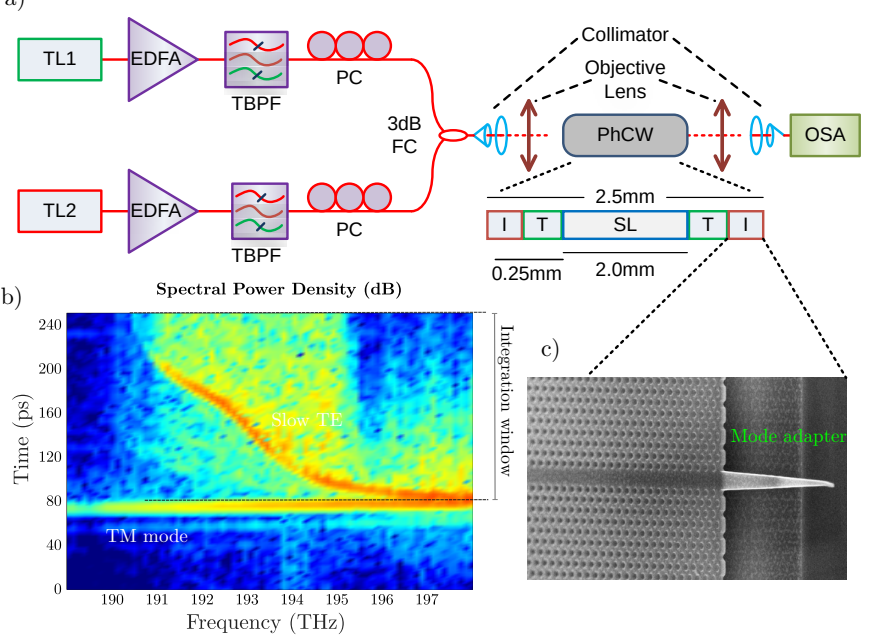

Fig. 1. (a) Experimental setup for the measurement of the FWM (TL=tunable laser, EDFA=erbium-doped fiber amplifier, $\mathrm{TBPF}=$ tunable bandpass filter, $\mathrm{PC}=$ polarization controller, $\mathrm{FC}=$ fiber coupler, OSA=optical spectrum analyzer) $\mathrm{PhC}$ waveguide sections. Inset: $\mathrm{PhC}$ waveguide sections with input (I), transition (T), and slow-light (SL) sections. (b) Density plot of the spectral power density transmitted depending on the propagation delay; the dynamic range of the logarithmic map is $70 \mathrm{~dB}$. (c) SEM image of the input section (mode adapter) of the waveguide.

tral distribution as a function of the transit time is represented in density plots (Fig.1(b)). There, the signature of the slow mode within the integration window is considered to extract the propagation attenuation of that specific mode and the transit time. The dispersion (Fig.2(a)-2(c)) is extracted from the measured arrival time and compared with calculations made assuming the nominal geometry (i.e. the e-beam layout) only allowing a slight change of the radius of the holes, which is process-dependent. This is carried out using an in-house implementation of the 3D finite-difference time-domain (FDTD) technique with the Floquet periodic boundary conditions and demonstrates very good agreement (Fig.2(a)-(c))) within measurement error. The group index $n_{g}$ over $\backsim 15 \mathrm{~nm}$ transmission window stays well above 13 and increases with wavelength. Group velocity dispersion (GVD) $\beta_{2}$ follows a trend typical of PhC waveguide, namely, it is negative (anomalous dispersion) and increases in magnitude to $-3 p s^{2} / m m$ and then reaches a local minimum. This dispersion profile is suitable for narrowband and broadband phase matching, thus allowing efficient parametric processes.

The insertion losses are deduced from the statistical analysis of 12 waveguides with varying lengths of the SL section (Fig.3(a)). The transmission is considered both in the "fast" $\left(n_{g} \approx 5\right)$ and in the "slow" $\left(n_{g} \approx 15\right)$ regime. Assuming that the attenuation mainly occurs in the SL section leads to an estimate of $50 \pm 20 \mathrm{~dB} / \mathrm{cm}$. In general, the estimate of the attenuation in the PhC in the slow-light regime is affected by considerable uncertainty, because of the inherently statistical nature of the disorder. The attenuation measured here is consistent with typical numbers in PhC waveguides [22]. The spectral dependence of the transmission (Fig.3(b)) is extracted from the integration in the time window indicated in the density plot (Fig.1(b)). In contrast with the raw transmission, this is representative of the fundamental TE mode, as the fine structure due to coherent scat-

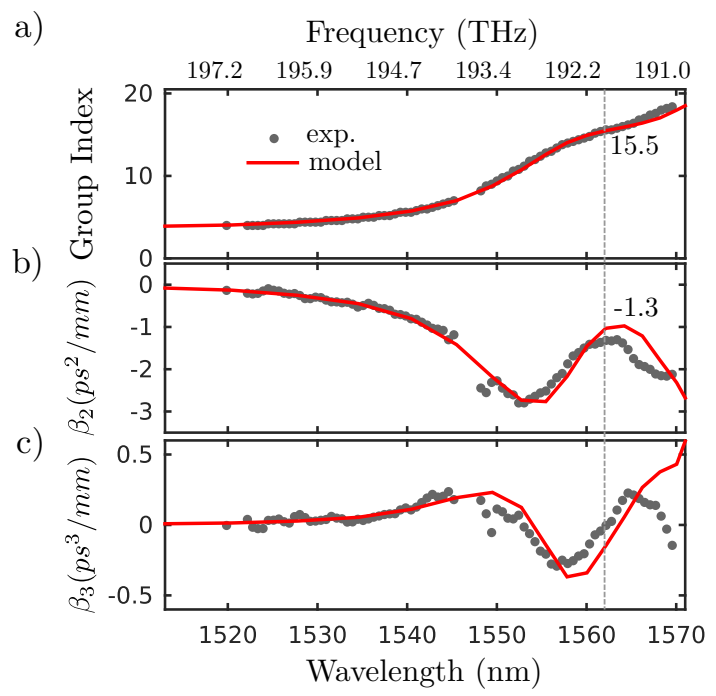

Fig. 2. Measured (circles) and calculated (solid line) (a) group index, (b) second and (c) third order dispersion. Values are given for $\lambda=1562 \mathrm{~nm}$.

tering $[23,24]$ and the interference with the TM mode are filtered out. Attenuation increases substantially $(>3 \mathrm{~dB})$ as $\lambda>1568 \mathrm{~nm}$.

Elastic scattering (= non absorbing) dominates the losses in these structures (which is common to $\mathrm{PhC}$ waveguides with transparent material). However, thermal effect are clearly observable even in the $\mathrm{CW}$ regime, where nonlinear absorption should be ruled out. Linear absorption, which could arise from surface defects in semiconductor, though much smaller than elastic scattering, can be measured indirectly through the thermorefractive effect that is known to be very sensitive [25]. Here we detect the spectral shift of the transmission fringes, which is directly related to the rise of the temperature due to absorption. A similar technique has already been used in the context of $\mathrm{PhC}$ waveguide [26]. The measurement is carried out with the setup in Fig.1(a) by combining an amplified pump with varied power with amplified spontaneous emission (ASE). The observed red spectral shift (Fig.3(c)) is linear with the on-chip power (here geometric average of the input and output levels), which clearly indicates linear residual absorption. The spectral shift is connected with the temperature of waveguide core through the coefficient where $d \lambda / d T=0.13 \pm 0.02 \mathrm{~nm} / \mathrm{K}$ which is obtained by rescaling the measurement $d \lambda / d T=0.09 \mathrm{~nm} / \mathrm{K}$ on similar PhC waveguides made of InGaP [27] with the ratio of the thermo-optic coefficients: $n_{T}(\mathrm{GaP}) / n_{T}(\operatorname{In} \mathrm{GaP})=1.5 \pm 0.2$ [28]. Then, the temperature is connected to the dissipation per unit length through the thermal conductivity (per unit waveguide length) $R_{t h, L}^{-1} \cong 1.0 \mathrm{~m}^{-1} \mathrm{~K}^{-1} \mathrm{~W}$, which is estimated based on the solution of heat diffusion equation with the COMSOL implementation of the finite element method. At last, the ratio with the on-chip power leads to the estimate for the linear absorption coefficient. The procedure is summarized by the formula:

$$
\alpha_{S L} L \cong \frac{\partial \lambda}{\partial P_{\text {in }}}\left(\frac{\partial \lambda}{\partial T} \cdot \frac{R_{t h}^{L}}{L}\right)^{-1}
$$

The absorption is estimated to $\alpha_{S L}=1.8 \mathrm{~dB} / \mathrm{cm}$ and the corresponding absorption rate is $\Gamma_{a b s}=\alpha_{a b s} v_{g}=2 \pi \times 130 \mathrm{MHz} \approx$ $8.2 \times 10^{8} \mathrm{~s}^{-1}$, which is consistent with independent measurements made on GaP suspended resonators [29]. We conjecture 
(a)

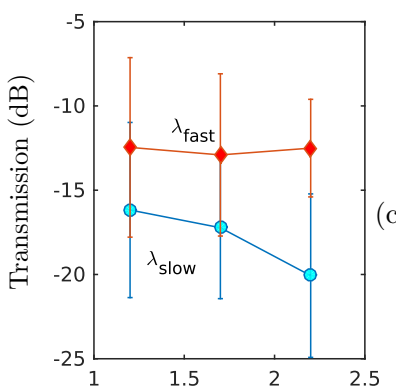

Slow Section Length $(\mathrm{mm})$

(b)

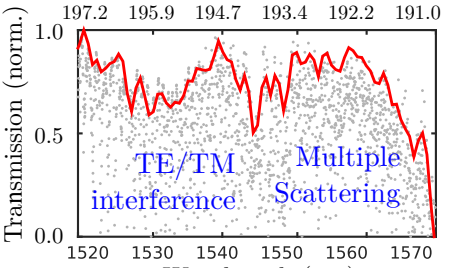

c)

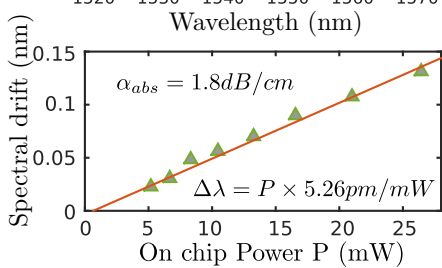

Fig. 3. (a) "Cut-back" measurement performed on 12 waveguides with varying length of the SL section. (b) Extracted transmission (norm.) from the density plot in Fig. 1b) (solid line) and raw transmission (points), evidencing scattering and interference. (e) Spectral shift of the transmission induced by the thermo-refractive effect as a function of the on-chip power. The corresponding absorption rate is $\Gamma_{\mathrm{abs}}=\alpha_{\mathrm{abs}} v_{\mathrm{g}}=$ $2 \pi \times 130 \mathrm{MHz} \approx 8.2 \times 10^{8} \mathrm{~s}^{-1}$.

that this result characterizes the residual absorption of nanoscale photonic structures made of $\mathrm{GaP}$ in the Telecom C-band, independently of the propagation/localization regime, to the extent the geometry (surface to volume ratio and spatial scale) is similar.

The ultra-fast Kerr response is measured through stimulated FWM under CW excitation (Fig.1(a)). To prevent unwanted nonlinear effects and to suppress ASE, the pump and probe, both polarized for the TE mode, are amplified independently by two erbium-doped fiber amplifiers (EDFAs) and successively filtered by tunable bandpass filters of $1 \mathrm{~nm}$ bandwidth before being combined by a 50:50 coupler. A representative measurement of the optical spectra at the output is shown in Fig.4(a) and 4(b), with on-chip power level at the waveguide input $P(0)=13.7 \mathrm{dBm}$, where one input is fixed at $1562 \mathrm{~nm}$ and the other spanning over $4 \mathrm{~nm}$, enough to reveal the phase mismatch. The nonlinear coefficient $\gamma$ is readily connected to the conversion efficiency of the stimulated FWM in the undepleted pump approximation: $\eta=P_{I}(L) / P_{B}(0)=\gamma^{2} P_{P}^{2}(0) L_{e f f}^{2}$ as long as $\gamma P_{P}(0) L_{e f f} \ll 1$, where $P_{m}(0)$ and $P_{m}(L)$, for $m=\{I, B, P\}$ represent the input and output pump, probe, and idler powers [30]. This is related, but not identical to the conversion efficiency $\eta_{L}=P_{I}(L) / P_{B}(L)$ obtained from the direct OSA readout. First $P(L)>P(0)$, because of losses. An additional difficulty arises from spectral irregularity of the transmission, which induces strong fluctuations in the estimate of the conversion efficiency.

The direct readout of the OSA has therefore to be corrected according to the extracted transmission.

$$
\eta=\eta_{L} e^{\left(\alpha_{I}-\alpha_{B}\right) L}=\gamma^{2} P_{P}(0)^{2} L_{e f f}^{2}
$$

having introduced the effective length $L_{e f f}=\left(1-e^{-\alpha L}\right) / \alpha$. The attenuation $\alpha$ is also moderately dispersive, following the extracted transmission. The result of the procedure is shown in Fig.4(c). The measured conversion efficiency now follows within about $\sigma=2 d B$ with the theoretical curve [31]:

$$
\eta=\eta_{0} \frac{\alpha^{2}}{\alpha^{2}+\Delta \beta^{2}}\left[1+4 \sin \left(\Delta \beta L_{e f f} / 2\right)^{2} \frac{\exp (-\alpha L)}{1-\exp (-2 \alpha L)}\right]
$$
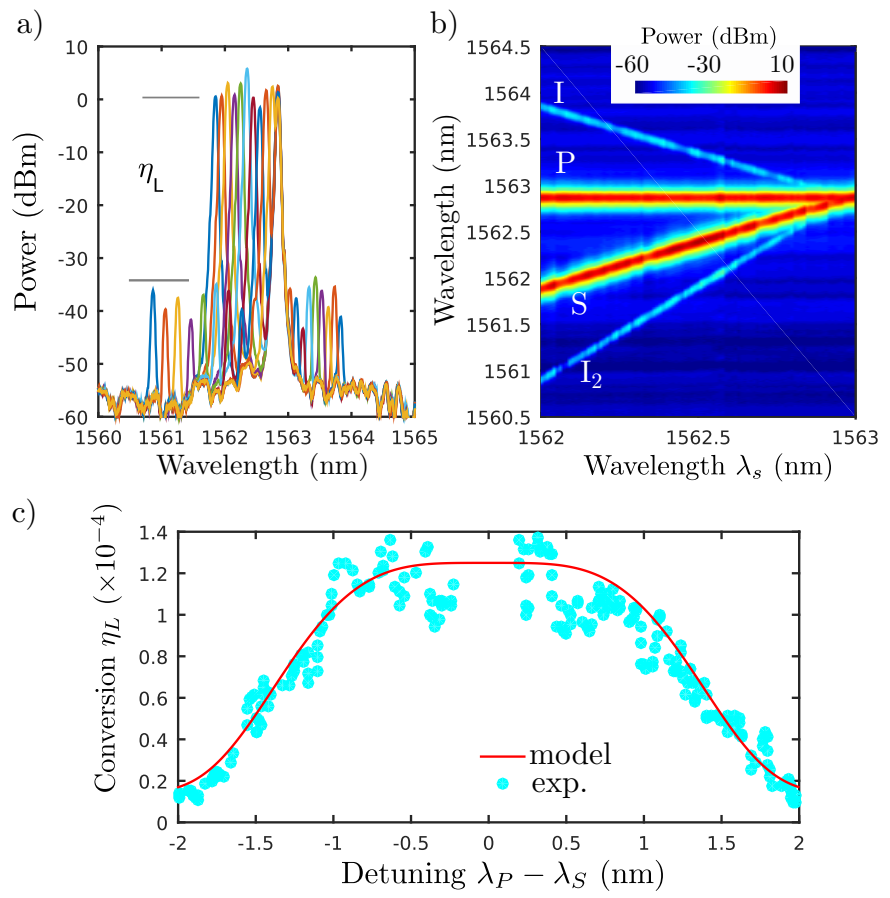

Fig. 4. FWM experiment. Direct OSA readout for $\lambda_{P}=$ $1562 \mathrm{~nm}$, fixed power and variable signal wavelength for some representative values (a) and the full measurement set represented as a density plot (b). (c) Extracted conversion efficiency vs. $\Delta \lambda$ with the theoretical phase matching calculated using dispersion from Fig.2(b).

where the $\Delta \beta$ is the wavevector mismatch extracted from the measured dispersion (Fig.2(b)) and the attenuation is about $\alpha=$ $1200 \mathrm{~m}^{-1}$ ( $\left.\approx 50 \mathrm{~dB} / \mathrm{cm}\right)$.

We now consider the conversion efficiency at phase matching, namely with a small detuning $|\Delta \lambda|<1 \mathrm{~nm}$. We remove "rare events" (values more than the $3 \mathrm{~dB}$ below the maximum) and consider the mean $\log \left(\bar{\eta}_{I}\right)=\left\langle\log \left(\eta_{I}\right)\right\rangle$ and standard deviation $\log \left(\sigma_{\eta}\right)=\left\langle\log \left(\eta_{I}^{2}\right)\right\rangle$. As the fluctuations are due to disorder induced resonances, we assume that to the first order their average corresponds to the expected conversion efficiency when resonant effects are off. Thus, we extract the nonlinear parameter $\gamma\left(\lambda_{P}\right)$. Changing input pump power while keeping pump and probe wavelengths fixed to $1562 \mathrm{~nm}$ and $1564 \mathrm{~nm}$ yields Fig.5(a) which is compared with the expected quadratic scaling (Eq. 2) and $\gamma\left(\lambda_{p, 0}\right)=(900 \pm 200) m^{-1} W^{-1}$.

The dispersion of the nonlinear response is measured under the conditions of Fig. 4 but changing the pump from 1560 to $1568 \mathrm{~nm}$ with $0.5 \mathrm{~nm}$ steps (Fig.5(b)). The error bars are representative of the uncertainty due to the fluctuations discussed earlier. The measurement is nevertheless consistent with the scaling of the so-called slow-down factor [18] and is summarized by the formula $\gamma\left(\lambda_{p}\right)=\gamma\left(\lambda_{p, 0}\right)\left(n_{g} / n_{g, 0}\right)^{2}$, with $\gamma\left(\lambda_{p, 0}\right)=(850 \pm 150) \mathrm{m}^{-1} \mathrm{~W}^{-1}$ at $\lambda_{P, 0}=1562 \mathrm{~nm}$ and $n_{g, 0}=15$. The uncertainty, hence the deviation from perfect scaling, increases with $n_{g}$, which is expected due to the increase of losses and disorder related effects as these abrupt losses are not entirely compensated in our procedure. These effects mask the correction to the $n_{g}^{2}$ scaling due to the spatial dependence of the mode [32], which is summarized in a nonlinear cross section $A_{\text {eff }}$ which is calculated using FDTD and represented by the black line in Fig.5(b). This leads to the extraction of the material 
a)

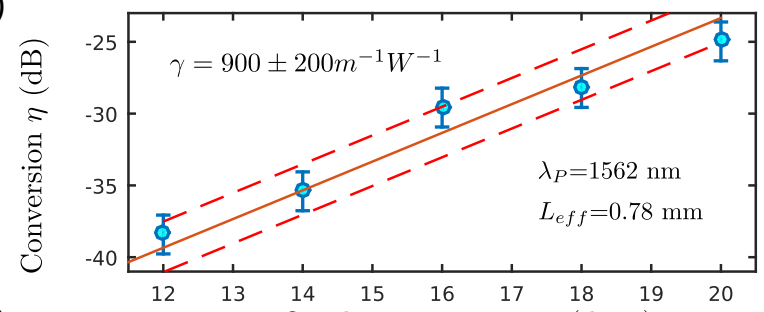

b)

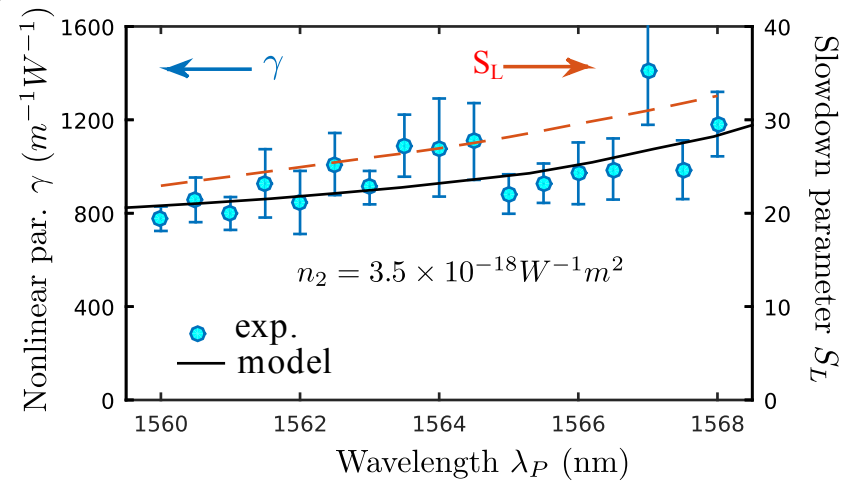

Fig. 5. (a) Conversion efficiency (symbols) vs. the on-chip pump power and fit (solid line) and confidence intervals (dashed). (b) Extracted nonlinear parameter $\gamma$ as a function of the pump wavelength (symbols) compared with the model (dashed line) and the slowdown scaling $S_{L}=n_{g}^{2} / n^{2}$.

nonlinearity $n_{2}=\left(\gamma \lambda A_{\text {eff }}\right) / 2 \pi=3.5 \times 10^{-18} \mathrm{~W}^{-1} \mathrm{~m}^{2}$.

In conclusion, we demonstrate efficient (up to $-25 \mathrm{~dB}$ ) parametric interactions in the $\mathrm{CW}$ regime in self-suspended $\mathrm{GaP}$ $\mathrm{SL}-\mathrm{PhC}$ waveguide. The FWM measurement is used to characterize the dispersive nonlinearity resulting from the waveguide and finally to extract an estimate of the material nonlinearity. Linear losses and residual absorption are also measured. More generally, we suggest that these results are representative of nonlinear nanoscale photonic structures, including metasurfaces as they take into account the effect of surface defects, residual absorption, disorder, and thermal effects which are common to structures with large surface to volume ratio. The propose measurement approach for the nonlinear interaction in nanoscale structures overcomes the difficulties inherent to the fabrication imperfections.

\section{FUNDING INFORMATION}

Labex (CONDOR); IDEX AAP IDI 2013 (37-2013); National Natural Science Foundation of China (NSFC) (61805014, 61827807, 61690193); This work was supported by the French RENATECH network.

\section{ACKNOWLEDGMENT}

The work of W. X., F. B., S. C., and A. D. R. is performed in the framework of the joint research lab between Thales Research \& Technology and Laboratoire Aimé Cotton.

\section{REFERENCES}

1. B. Kuyken, H. Ji, S. Clemmen, S. K. Selvaraja, H. Hu, M. Pu, M. Galili, P. Jeppesen, G. Morthier, S. Massar et al., Opt. express 19, B146 (2011).
2. H. Hu, E. Palushani, M. Galili, H. C. H. Mulvad, A. Clausen, L. K. Oxenløwe, and P. Jeppesen, Opt. express 18, 9961 (2010).

3. J. S. Levy, A. Gondarenko, M. A. Foster, A. C. Turner-Foster, A. L. Gaeta, and M. Lipson, Nat. photonics 4, 37 (2010).

4. M. Pu, L. Ottaviano, E. Semenova, and K. Yvind, Optica 3, 823 (2016).

5. B. Jalali and S. Fathpour, J. Light. Technol. 24, 4600 (2006).

6. E. W. Van Stryland, H. Vanherzeele, M. A. Woodall, M. Soileau, A. L. Smirl, S. Guha, and T. F. Boggess, Opt. Eng. 24, 244613 (1985).

7. K. Nozaki, A. Shinya, S. Matsuo, Y. Suzaki, T. Segawa, T. Sato, Y. Kawaguchi, R. Takahashi, and M. Notomi, Nat. Photonics 6, 248 (2012).

8. J. S. Aitchison, D. C. Hutchings, J. U. Kang, G. I. Stegeman, and A. Villeneuve, IEEE J. Quantum Electron. 33, 341 (1997).

9. P. Colman, C. Husko, S. Combrié, I. Sagnes, C. W. Wong, and A. De Rossi, Nat. Photonics 4, 862 (2010).

10. K. Rivoire, S. Buckley, and J. Vuckovic, Opt. Express 19, 22198 (2011).

11. M. Mitchell, A. C. Hryciw, and P. E. Barclay, Appl. Phys. Lett. 104, 141104 (2014).

12. D. P. Lake, M. Mitchell, H. Jayakumar, L. F. dos Santos, D. Curic, and P. E. Barclay, Appl. Phys. Lett. 108, 031109 (2016).

13. K. Rivoire, Z. Lin, F. Hatami, W. T. Masselink, and J. Vuckovic, Opt. Express 17, 22609 (2009).

14. D. J. Wilson, K. Schneider, S. Hoenl, M. Anderson, T. J. Kippenberg, and P. Seidler, arXiv preprint arXiv:1808.03554 (2018).

15. A. Martin, S. Combrié, A. de Rossi, G. Beaudoin, I. Sagnes, and F. Raineri, Photonics Res. 6, B43 (2018).

16. A. Bilenca, R. Alizon, V. Mikhelashhvili, D. Dahan, G. Eisenstein, R. Schwertberger, D. Gold, J. Reithmaier, and A. Forchel, IEEE Photonics Technol. Lett. 15, 563 (2003).

17. Q. V. Tran, S. Combrié, P. Colman, and A. D. Rossi, Appl. Phys. Lett. 95, 061105 (2009).

18. C. Monat, M. De Sterke, and B. Eggleton, J. Opt. 12, 104003 (2010).

19. A. Parini, P. Hamel, A. De Rossi, S. Combrie, N. Tran, Y. Gottesman, R. Gabet, A. Talneau, Y. Jaouen, and G. Vadala, J. Light. Technol. 26, 3794 (2008).

20. C. Caër, S. Combrié, X. Le Roux, E. Cassan, and A. De Rossi, Appl. Phys. Lett. 105, 121111 (2014).

21. S. Combrié, G. Lehoucq, G. Moille, A. Martin, and A. De Rossi, Laser \& Photonics Rev. 11, 1700099 (2017).

22. L. O'Faolain, S. A. Schulz, D. M. Beggs, T. P. White, M. Spasenović, L. Kuipers, F. Morichetti, A. Melloni, S. Mazoyer, J.-P. Hugonin et al., Opt. express 18, 27627 (2010).

23. M. Patterson, S. Hughes, S. Combrié, N.-V.-Q. Tran, A. De Rossi, R. Gabet, and Y. Jaouën, Phys. review letters 102, 253903 (2009).

24. S. Mazoyer, J.-P. Hugonin, and P. Lalanne, Phys. review letters 103, 063903 (2009).

25. A. Boccara, D. Fournier, and J. Badoz, Appl. Phys. Lett. 36, 130 (1980).

26. A. Martin, D. Sanchez, S. Combrié, A. de Rossi, and F. Raineri, Opt. Lett. 42, 599 (2017).

27. S. Sokolov, J. Lian, S. Combrié, A. D. Rossi, and A. P. Mosk, Appl. Opt. 56, 3219 (2017).

28. $n_{T}(\mathrm{GaP}) \approx 3 \times 10^{-4} \mathrm{~K}^{-1}$ is from the database of the loffe Instut http://www.ioffe.ru/SVA/NSM/Semicond/, while $n_{T}(\operatorname{InGaP})$ is from ref. [27].

29. I. Ghorbel, F. Swiadek, R. Zhu, D. Dolfi, G. Lehoucq, A. Martin, G. Moille, L. Morvan, R. Braive, S. Combrié, and A. D. Rossi, arXiv preprint arXiv:1901.05922 (2019).

30. M. Ebnali-Heidari, C. Monat, C. Grillet, and M. K. Moravvejfarshi, Opt. Express 17, 18340 (2009).

31. P. P. Absil, J. V. Hryniewicz, B. E. Little, P. S. Cho, R. A. Wilson, L. G. Joneckis, and P.-T. Ho, Opt. Lett. 25, 554 (2000).

32. M. Santagiustina, C. G. Someda, G. Vadala, S. Combrié, and A. De Rossi, Opt. Express 18, 21024 (2010). 


\section{FULL REFERENCES}

1. B. Kuyken, H. Ji, S. Clemmen, S. K. Selvaraja, H. Hu, M. Pu, M. Galili, P. Jeppesen, G. Morthier, S. Massar et al., "Nonlinear properties of and nonlinear processing in hydrogenated amorphous silicon waveguides," Opt. express 19, B146-B153 (2011).

2. H. Hu, E. Palushani, M. Galili, H. C. H. Mulvad, A. Clausen, L. K. Oxenløwe, and P. Jeppesen, "640 gbit/s and $1.28 \mathrm{tbit} / \mathrm{s}$ polarisation insensitive all optical wavelength conversion," Opt. express 18, 99619966 (2010).

3. J. S. Levy, A. Gondarenko, M. A. Foster, A. C. Turner-Foster, A. L. Gaeta, and M. Lipson, "Cmos-compatible multiple-wavelength oscillator for on-chip optical interconnects," Nat. photonics 4, 37 (2010).

4. M. Pu, L. Ottaviano, E. Semenova, and K. Yvind, "Efficient frequency comb generation in algaas-on-insulator," Optica 3, 823-826 (2016).

5. B. Jalali and S. Fathpour, "Silicon photonics," J. Light. Technol. 24, 4600-4615 (2006).

6. E. W. Van Stryland, H. Vanherzeele, M. A. Woodall, M. Soileau, A. L. Smirl, S. Guha, and T. F. Boggess, "Two photon absorption, nonlinear refraction, and optical limiting in semiconductors," Opt. Eng. 24, 244613 (1985).

7. K. Nozaki, A. Shinya, S. Matsuo, Y. Suzaki, T. Segawa, T. Sato, Y. Kawaguchi, R. Takahashi, and M. Notomi, "Ultralow-power all-optical ram based on nanocavities," Nat. Photonics 6, 248 (2012).

8. J. S. Aitchison, D. C. Hutchings, J. U. Kang, G. I. Stegeman, and A. Villeneuve, "The nonlinear optical properties of algaas at the half band gap," IEEE J. Quantum Electron. 33, 341-348 (1997).

9. P. Colman, C. Husko, S. Combrié, I. Sagnes, C. W. Wong, and A. De Rossi, "Temporal solitons and pulse compression in photonic crystal waveguides," Nat. Photonics 4, 862 (2010).

10. K. Rivoire, S. Buckley, and J. Vuckovic, "Multiply resonant photonic crystal nanocavities for nonlinear frequency conversion," Opt. Express 19, 22198-22207 (2011).

11. M. Mitchell, A. C. Hryciw, and P. E. Barclay, "Cavity optomechanics in gallium phosphide microdisks," Appl. Phys. Lett. 104, 141104 (2014).

12. D. P. Lake, M. Mitchell, H. Jayakumar, L. F. dos Santos, D. Curic, and P. E. Barclay, "Efficient telecom to visible wavelength conversion in doubly resonant gallium phosphide microdisks," Appl. Phys. Lett. 108, 031109 (2016).

13. K. Rivoire, Z. Lin, F. Hatami, W. T. Masselink, and J. Vuckovic, "Second harmonic generation in gallium phosphide photonic crystal nanocavities with ultralow continuous wave pump power," Opt. Express 17, 2260922615 (2009).

14. D. J. Wilson, K. Schneider, S. Hoenl, M. Anderson, T. J. Kippenberg, and P. Seidler, "Gallium phosphide nonlinear photonics," arXiv preprint arXiv:1808.03554 (2018).

15. A. Martin, S. Combrié, A. de Rossi, G. Beaudoin, I. Sagnes, and F. Raineri, "Nonlinear gallium phosphide nanoscale photonics," Photonics Res. 6, B43-B49 (2018).

16. A. Bilenca, R. Alizon, V. Mikhelashhvili, D. Dahan, G. Eisenstein, R. Schwertberger, D. Gold, J. Reithmaier, and A. Forchel, "Broad-band wavelength conversion based on cross-gain modulation and four-wave mixing in inas-inp quantum-dash semiconductor optical amplifiers operating at $1550 \mathrm{~nm}$," IEEE Photonics Technol. Lett. 15, 563-565 (2003).

17. Q. V. Tran, S. Combrié, P. Colman, and A. D. Rossi, "Photonic crystal membrane waveguides with low insertion losses," Appl. Phys. Lett. 95, 061105 (2009).

18. C. Monat, M. De Sterke, and B. Eggleton, "Slow light enhanced nonlinear optics in periodic structures," J. Opt. 12, 104003 (2010).

19. A. Parini, P. Hamel, A. De Rossi, S. Combrie, N. Tran, Y. Gottesman, R. Gabet, A. Talneau, Y. Jaouen, and G. Vadala, "Time-wavelength reflectance maps of photonic crystal waveguides: A new view on disorder-induced scattering," J. Light. Technol. 26, 3794-3802 (2008).

20. C. Caër, S. Combrié, X. Le Roux, E. Cassan, and A. De Rossi, "Extreme optical confinement in a slotted photonic crystal waveguide," Appl. Phys. Lett. 105, 121111 (2014).

21. S. Combrié, G. Lehoucq, G. Moille, A. Martin, and A. De Rossi, "Comb of high-q resonances in a compact photonic cavity," Laser \& Photonics Rev. 11, 1700099 (2017).
22. L. O'Faolain, S. A. Schulz, D. M. Beggs, T. P. White, M. Spasenović, L. Kuipers, F. Morichetti, A. Melloni, S. Mazoyer, J.-P. Hugonin et al., "Loss engineered slow light waveguides," Opt. express 18, 2762727638 (2010).

23. M. Patterson, S. Hughes, S. Combrié, N.-V.-Q. Tran, A. De Rossi, R. Gabet, and Y. Jaouën, "Disorder-induced coherent scattering in slowlight photonic crystal waveguides," Phys. review letters 102, 253903 (2009).

24. S. Mazoyer, J.-P. Hugonin, and P. Lalanne, "Disorder-induced multiple scattering in photonic-crystal waveguides," Phys. review letters $\mathbf{1 0 3}$ 063903 (2009).

25. A. Boccara, D. Fournier, and J. Badoz, "Thermo-optical spectroscopy: Detection by the"mirage effect”," Appl. Phys. Lett. 36, 130-132 (1980).

26. A. Martin, D. Sanchez, S. Combrié, A. de Rossi, and F. Raineri, "Gainp on oxide nonlinear photonic crystal technology," Opt. Lett. 42, 599-602 (2017).

27. S. Sokolov, J. Lian, S. Combrié, A. D. Rossi, and A. P. Mosk, "Measurement of the linear thermo-optical coefficient of ga0.51in $0.49 \mathrm{p}$ using photonic crystal nanocavities," Appl. Opt. 56, 3219-3222 (2017).

28. $n_{T}(\mathrm{GaP}) \approx 3 \times 10^{-4} \mathrm{~K}^{-1}$ is from the database of the loffe Instut http://www.ioffe.ru/SVA/NSM/Semicond/, while $n_{T}(\operatorname{InGaP})$ is from ref. [27].

29. I. Ghorbel, F. Swiadek, R. Zhu, D. Dolfi, G. Lehoucq, A. Martin, G. Moille, L. Morvan, R. Braive, S. Combrié, and A. D. Rossi, "Optomechanical gigahertz oscillator made of a two photon absorption free piezoelectric iii/v semiconductor," arXiv preprint arXiv:1901.05922 (2019).

30. M. Ebnali-Heidari, C. Monat, C. Grillet, and M. K. Moravvejfarshi, "A proposal for enhancing four-wave mixing in slow light engineered photonic crystal waveguides and its application to optical regeneration," Opt. Express 17, 18340-18353 (2009).

31. P. P. Absil, J. V. Hryniewicz, B. E. Little, P. S. Cho, R. A. Wilson, L. G. Joneckis, and P.-T. Ho, "Wavelength conversion in gaas micro-ring resonators," Opt. Lett. 25, 554-556 (2000).

32. M. Santagiustina, C. G. Someda, G. Vadala, S. Combrié, and A. De Rossi, "Theory of slow light enhanced four-wave mixing in photonic crystal waveguides," Opt. Express 18, 21024-21029 (2010). 